\title{
PROSPECTS FOR DETECTING UNSEEN COMPANIONS OF PERIODIC VARIABLES
}

\author{
A. PIGULSKI \\ Wroctaw University Observatory, \\ Kopernika 11, 51-622 Wroctaw, Poland
}

\begin{abstract}
All periodic variables with stable periods such as pulsating stars, eclipsing binaries and pulsars offer the possibility of detecting unseen companion(s) by means of the light-time effect. We discuss the limitations of the method (the visibility of the effect) for different types of periodic variables. Special attention is paid to the ranges of mass ratios and orbital periods in which unseen companions can be found. We also indicate several systems with light-time effect in which hypothetical companions can be detected by speckle interferometry or precise astrometric observations. In these cases, the detection of the companions may lead to the determination of the components' masses.
\end{abstract}

\section{Introduction}

Any object emitting periodic signal is a candidate for the detection of the light-time effect (hereafter LTE) provided that it belongs to a binary or multiple system. This effect causes apparent period changes in accordance to the motion along the binary orbit. By the detection of LTE in period changes of a periodic variable, the presence of its unseen companion can be uncovered. Independently of the fact whether the companion is observed directly or not, the study of LTE may give an important contribution to our knowledge of the binary.

\section{Limitations of the method}

A periodic variable used to the study the LTE should have a stable period. ¿From all known periodic variables, some types of pulsating stars, eclipsing 
binaries, and pulsars have enough stable periods to be promising candidates for the study of LTE.

In order to estimate the visibility of the effect in the O-C diagram, let us consider-for simplicity-LTE in a double system with a circular orbit. In the O-C diagram, the changes of the period $P$ of the signal emitted by the visible component due to LTE will have the form of a sine curve with semi-amplitude equal to $P_{\text {orb }} K_{\text {vis }} /(2 \pi c)$, where $P_{\text {orb }}$ is the orbital period, and $2 K_{\text {vis }}$ is the range of the primary's radial-velocity curve.

The amplitude of LTE depents proportionally on $P_{\text {orb }}$ and $K_{\text {vis }}$ and is equal to the time which light needs for passing the projected orbit. The LTE can be detected only if this amplitude is larger than the typical error of the determination of the phase of periodic signal. Since this error determines the scatter of points in the O-C diagram, the visibility of LTE will, if fact, depend on the $P_{\text {orb }} / P$ ratio. Consequently, for a given orbital period, short-period variables are more suitable for the study of LTE than the longperiod ones. Furthermore, the amplitude of the effect is proportional to the mass ratio $q$ (defined as $M_{\text {unseen }} / M_{\text {visible }}$ ). Assuming that the range of the variation due to LTE is larger than $0.02 P$, we estimated the minimum mass ratios for different types of periodic variables (see Table 1). In the $P_{\text {orb }}-q$ plane, shown in Fig. 1, we present the lower limits for the detection of LTE for all periodic variables listed in Table 1 .

TABLE 1. Minimum mass ratios $q$ and minimum masses of unseen companions which can be found by means of LTE in a system with $P_{\text {orb }}=100 \mathrm{yr}$. Minimum $P_{\text {orb }}$ needed for the detection of a $1 M_{\odot}$ companion is given in the last column.

\begin{tabular}{cccccc}
\hline $\begin{array}{c}\text { Type of visible } \\
\text { object }\end{array}$ & $\begin{array}{c}\text { Typical } \\
\text { mass }\end{array}$ & $\begin{array}{c}\text { Typical } \\
\text { period }\end{array}$ & $\begin{array}{c}\text { Minimum } \\
q\end{array}$ & $\begin{array}{c}\text { Minimum } \\
M_{\text {unseen }}\end{array}$ & $\begin{array}{c}\text { Minimum } \\
P_{\text {orb }}\end{array}$ \\
\hline RR Lyrae star & $0.6 M_{\odot}$ & $0.5 \mathrm{~d}$ & 0.05 & $0.03 M_{\odot}$ & $1.3 \mathrm{yr}$ \\
Classical Cepheid & $8 M_{\odot}$ & $8 \mathrm{~d}$ & 0.4 & $3 M_{\odot}$ & $500 \mathrm{yr}$ \\
$\beta$ Cephei star & $12 M_{\odot}$ & $0.2 \mathrm{~d}$ & 0.007 & $0.08 M_{\odot}$ & $2.5 \mathrm{yr}$ \\
$\delta$ Scuti star & $2 M_{\odot}$ & $2 \mathrm{~h}$ & 0.005 & $0.01 M_{\odot}$ & $60 \mathrm{~d}$ \\
pulsating WD & $1 M_{\odot}$ & $500 \mathrm{~s}$ & 0.0005 & $0.5 M_{\text {Jupiter }}$ & $1 \mathrm{~d}$ \\
\hline eclipsing binary & $3 M_{\odot}$ & $2 \mathrm{~d}$ & 0.06 & $0.2 M_{\odot}$ & $9 \mathrm{yr}$ \\
\hline typical pulsar & $1.5 M_{\odot}$ & $1 \mathrm{~s}$ & $10^{-6}$ & $0.5 M_{\text {Earth }}$ & no limit \\
millisecond pulsar & $1.5 M_{\odot}$ & $5 \mathrm{~ms}$ & $4 \times 10^{-9}$ & $0.2 M_{\text {Moon }}$ & no limit \\
\hline
\end{tabular}

As one can see from Fig. 1, the shorter is the period of the signal the less massive companions can be discovered by means of LTE. In the systems containing pulsars or pulsating white dwarfs, there is a possibility that planetary companion(s) will be discovered. 


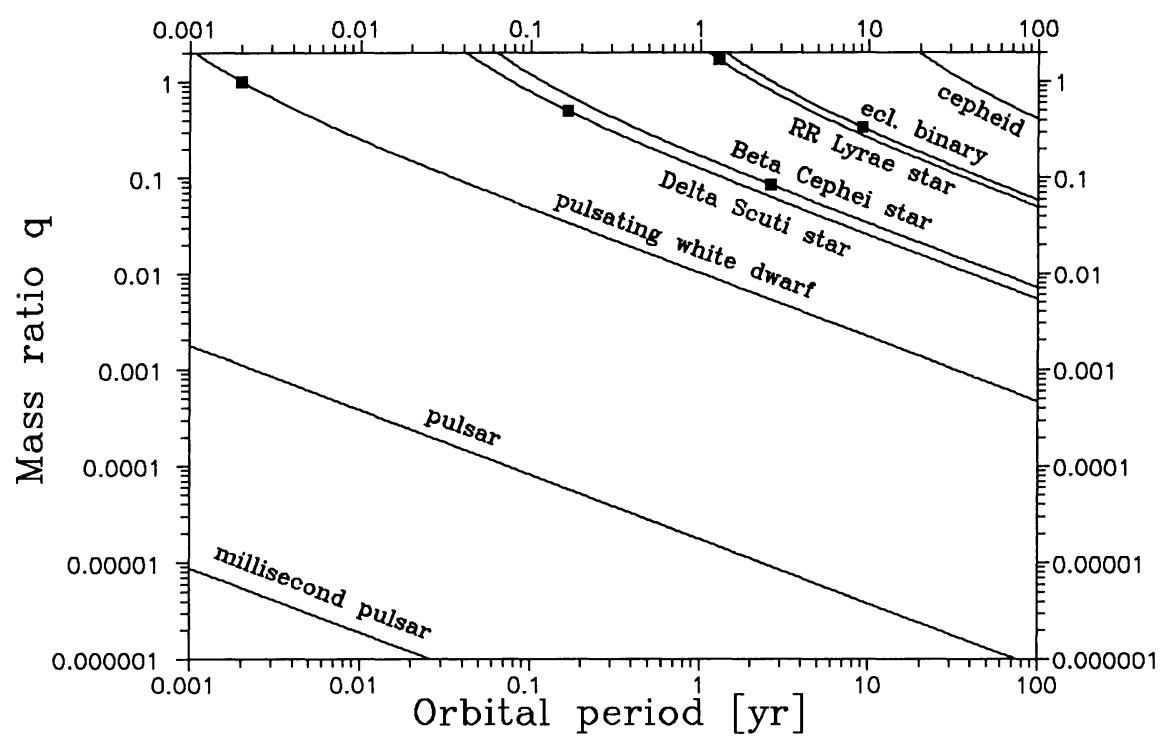

Figure 1. The lower limits for the detection of LTE for several types of periodic variables. A circular orbit and the most favourable inclination $\left(i=90^{\circ}\right)$ were assumed. The filled squares indicate the case of a $1 M_{\odot}$ companion.

\section{Conclusions and examples}

Apart from the discovery of the presence of an unseen companion, the detection of LTE can yield another result: a spectroscopic orbit can be found from the O-C diagram if the observations cover more than one orbital cycle. This is very important in the case of pulsating variables, the radial velocities of which are strongly affected by the pulsation.

If the presence of a LTE companion (i.e., companion discovered by means of LTE) can be confirmed by other independent observations this can yield additional information about the system. The high-quality spectrographic observations can reveal the secondary spectrum or provide radialvelocity curve of the primary (it should be consistent with the observed LTE). On the other hand, some LTE companions (if not very faint in comparison with the primary) can be resolved by interferometric methods yielding a relative visual orbit. Next, the absolute visual orbit can be obtained with the precise astrometric observations. This is very important because a combination of spectroscopic and visual (astrometric/speckle) orbits can lead to the determination of the masses of the components as well as other astrophysical parameters of the system.

With this in mind, we prepared a somewhat arbitrary list of objects (Table 2) which probably have companions because quite convincing pe- 
riodic changes of their periods-presumably due to LTE-are observed. Independent confirmation of the presence of a companion for any of these objects would constitute an interesting and important contribution to their study.

This work was supported by the IAU grant and the reasearch KBN grant Nr 2 P304 00104.

TABLE 2. Some periodic variables for which period changes attributable to LTE are observed.

\begin{tabular}{lcccl}
\hline Variable name & Type & $P[\mathrm{~d}]$ & $P_{\text {orb }}[\mathrm{yr}]$ & Reference \\
\hline RX Aur & cepheid & 11.624 & 53.8 & Szabados (1988) \\
IU Aur & EB & 1.812 & 0.81 & Mayer \& Drechsel (1987) \\
AH Cep & EB & 1.775 & 62.9 & Drechsel et al. (1989) \\
FZ CMa & EB & 1.273 & 1.47 & Moffat et al. (1983) \\
IM Aur & EB & 1.247 & 3.78 & Bartolini \& Zoffali (1986) \\
TU UMa & RR Lyr & 0.558 & 23 & Szeidl et al. (1986) \\
BW Vul & $\beta$ Cep & 0.201 & 33.5 & Pigulski (1993) \\
SZ Lyn & $\delta$ Sct & 0.121 & 3.22 & Paparó et al. (1988) \\
KZ Hya & $\delta$ Sct & 0.060 & 9.3 & Liu et al. (1991) \\
PSR 1820-11 & pulsar & $0.28 \mathrm{~s}$ & 0.98 & Phinney \& Verbunt (1991) \\
\hline
\end{tabular}

\section{References}

Bartolini C. and Zoffali M. (1986) Light-time effect in IM Aurigae, Astron. E Astroph., 168, pp. 377-379

Drechsel H., Lorenz R. and Mayer P. (1989) Solution of the light curves with third light contribution: the eclipsing binaries LY Aurigae and $\mathrm{AH}$ Cephei reconsidered, Astron. \& Astroph., 221, pp. 49-61

Liu Y., Jiang S. and Cao M. (1991) A further proof on the binary model of HD 94033, $I B V S$ No. 3606

Mayer P. and Drechsel H. (1987) Up-to-date parameters of the eclipsing triple system IU Aur, Astron. \& Astroph., 183, pp. 61-65

Moffat A.F.J., Vogt N., Vaz L.P.R. and Grønbech B. (1983) The early B-type eclipsing binary FZ CMa (HD 52942): a masive triple system, Astron. \& Astroph., 120, pp. $278-286$

Paparó M., Szeidl B. and Mahdy H.A. (1988) The high amplitude Delta Scuti star SZ Lyncis revisited, Space Science Review, 149, pp. 73-82

Phinney E.S. and Verbunt F. (1991) Binary pulsars before spin-up and PSR 1820-11, Monthly Not. Royal Astron. Soc., 248, pp. 21P-23P

Pigulski A. (1993) The light-time effect as the cause of period changes in $\beta$ Cephei stars. III. BW Vulpeculae, Astron. \& Astroph., 274, pp. 269-273

Szabados L. (1988) Possible light-time effect in the cepheids FN Aquilae and RX Aurigae, Publ. Astron. Soc. Pacific, 100, pp. 589-593

Szeidl B., Oláh K. and Mizser A. (1986) Period changes of RR Lyrae stars II, TW Her, VZ Her, AV Peg and TU UMa, Comm. Konkoly Obs. No. 89, pp. 57-110 